\title{
Sagittal Abdominal Diameter does not Predict Metabolic Traits Better than Waist Circumference-Related Measures of Abdominal Obesity in Obese Subjects
}

Authors

Runa Zazai1, Britta Wilms², Barbara Ernst ${ }^{3}$, Rahel Keppler ${ }^{1}$, Martin Thurnheer ${ }^{3}$, Sebastian M. Schmid², Bernd Schultes ${ }^{3}$

Affiliations

1 Department of Surgery, Cantonal Hospital St. Gallen, St.Gallen, Switzerland

2 Department of Internal Medicine I, University Hospital Lübeck and German Center for Diabetes Research (DZD), Lübeck, Germany

3 eSwiss Medical \& Surgical Center, St. Gallen, Switzerland

\section{Key words}

waist circumference, sagittal abdominal diameter, glucose, lipids, uric acid, metabolic syndrome

received $\quad 15.05 .2017$

revised 19.09.2017

accepted 16.10.2017

\section{Bibliography}

DOI https://doi.org/10.1055/s-0043-121568

Published online: 21.12.2017

Exp Clin Endocrinol Diabetes 2018; 126: 619-627

(C) J. A. Barth Verlag in Georg Thieme Verlag KG Stuttgart .

New York

ISSN 0947-7349

\section{Correspondence}

Prof. Dr. med. Bernd Schultes

eSwiss Medical \& Surgical Center

Brauerstrasse 97

9016 St. Gallen

Switzerland

Tel.: +4171282 2050, Fax: +4171282 2059

bernd.schultes@eswiss.center

\section{ABSTRACT}

Background Recently, we showed that in subjects with a body mass index $(\mathrm{BMI})>35 \mathrm{~kg} / \mathrm{m}^{2}$ waist circumference (WC) is associated with metabolic traits but associations were weaker in men than in women.

Aims To confirm our previous observation that anthropometric measures of abdominal obesity are closer linked to metabolic traits in obese women than obese men. To test whether sagittal abdominal diameter (SAD) provides a better prediction of metabolic traits in obese subjects than WC related measures. Methods SAD and WC along with metabolic traits were assessed in 204 women and 69 men (BMI $30.1-64.0 \mathrm{~kg} / \mathrm{m}^{2}$ ).

Results In women, abdominal obesity measures were associated with glycated hemoglobin levels (HbA1c), fasting serum glucose, insulin, triglycerides (TG), total cholesterol (Chol), high-density lipoprotein (HDL), and uric acid levels as well as Chol/HDL ratio and homeostatic model assessment of insulin resistance (HOMA-IR) independently of age and BMI. SAD predicted serum insulin and Chol better than WC, whereas WC was superior to SAD in predicting HbA1c, glucose, HOMA-IR, TG, $\mathrm{HDL}$, Chol/HDL, and uric acid. Of note, the combination of SAD and WC provided a better prediction of insulin, HOMA-IR, TG, and uric acid than each of these anthropometric alone. In men, only fasting glucose, Chol, and uric acid levels were associated with abdominal obesity markers.

Conclusion Data show various associations between anthropometric measures of abdominal obesity and metabolic traits in obese women but overall much less in obese men. Most metabolic traits are better predicted by WC than by SAD.

\section{Introduction}

Obesity is associated with a plethora of adverse metabolic traits such disturbed glucose metabolism, dyslipidemia, and hypertension often referred to as the metabolic syndrome. Apart from the overall degree of obesity, which is reflected by the body mass index (BMI), body fat distribution plays an important role in determining metabolic health and cardiovascular risk [1, 2]. In particular, abdominal obesity has been found to be associated with adverse metabolic traits and cardiovascular outcomes [3-6].
The most accurate methods to measure abdominal obesity and visceral adiposity in particular are computed tomography (CT) and magnetic resonance imaging (MRI). However, these methods are expensive and not widely available [7]. Therefore, several anthropometric measures such as waist circumference (WC) and related indices such as WC related to height (W/Ht ratio) or hip circumference (waist/hip ratio, WHR) have been established as surrogate markers of abdominal obesity [8]. Many studies have shown that these anthropometric measures, in fact, predict adverse metabolic traits and health outcomes much better than the BMI alone [4-6, 9-19]. 
In subjects with obesity, as defined by an BMI above $30 \mathrm{~kg} / \mathrm{m}^{2}$, the role of WC and related anthropometric measures of abdominal obesity is less clear since most of affected subjects already display a WC that is greater than the suggested cut-off points for the prediction of an increased health risk [8]. Putting this forward, we have previously performed a cross-sectional study in which we measured WC in 838 obese subjects ( 597 women) with a BMI of greater than $35 \mathrm{~kg} / \mathrm{m}^{2}$, i. e., grade 2 or higher obesity, along with cardiometabolic risk markers such as blood pressure, glucose, insulin, lipid and uric acid levels [20]. Multivariate regression analyses of respective data revealed that WC as well as related anthropometric indices, in particular those accounting for subjects' height, were associated with many metabolic variables independently of body weight and BMI. Another interesting finding in this study was that WC-related indices were more closely associated with metabolic traits in women than in men. This suggests that abdominal fat accumulation, as indicated by increased WC, plays an important role in women displaying grade 2 or higher obesity.

The landmarks required for a proper measurement of WC, i. e., the lower margin of the least palpable rib and the top of the iliac crest [8], are often not easy to determine in obese subjects, which makes a reliable and accurate assessment of WC very difficult. The sagittal abdominal diameter (SAD) may represent a valuable alternative to WC since it appears to be much easier to assess with a higher reliability. Several studies have shown that SAD measurement can help to predict adverse metabolic traits and cardiovascular outcomes in distinct populations [21-30]. In obese subjects, SAD may even closer reflect the degree of visceral adiposity since it is measured in a supine position were accumulated subcutaneous abdominal fat layers, which are believed to be metabolically much less harmful, partly slide to the lateral sides and thus, do not impact the measure as much as WC [31]. However, to the best of our knowledge the value of SAD in predicting metabolic trait specifically in generally obese subjects has not been assessed so far.

The aim of our present study was 1) to confirm, in an independent sample, our previous observation that WC-related anthropometric measurers of abdominal obesity are independently of body weight and BMI associated with metabolic traits in subjects with a wide range of obesity, 2) to confirm that abdominal obesity markers in generally obese subjects are stronger associated with metabolic traits in obese women than men, and 3) to test the hypothesis that SAD is superior to WC-related measures in predicting metabolic traits in obese subjects.

\section{Materials and Methods}

\section{Subjects}

This was a cross-sectional study for which we extracted data from 204 obese women and 69 obese men from our prospectively maintained database. All subjects were examined between March 2011 and April 2012 and were referred to our center specifically for obesity evaluation and treatment. Data were collected as a part of our standardized clinical evaluation protocol. Inclusion criteria for the study were a BMI greater than $30.0 \mathrm{~kg} / \mathrm{m}^{2}$ and an age of at least 18 years. Exclusion criteria was the inclusion of subject's data set in previously published data analyses on the association between anthro- pometric indices and metabolic traits [20]. All subjects gave written informed consent for scientific use of their clinical data and the study was carried out in accordance with the recommendations of the Swiss Federal Expert Commission for Physician Confidentiality.

\section{Anthropometric measurement}

Body weight was measured to the nearest $0.1 \mathrm{~kg}$ with subjects wearing light clothes and height was measured to the nearest $0.5 \mathrm{~cm}$. BMI were calculated by weight in kilograms divided by height in meters squared. WC was measured to the nearest $0.5 \mathrm{~cm}$ midway between the lowest rib and the iliac crest while subject was at minimal respiration according to World Health Organization [8]. In cases in which the respective anatomic landmarks were not identifiable, the maximal WC was measured. Hip circumference (HC) was measured around the widest portion of the buttocks [8]. Upon these measurements waist to hip ratio (WHR) and Waist to height ratio $(\mathrm{W} / \mathrm{Ht}$ ) were calculated. SAD was measured by using a caliper (Holtain-Kahn Abdominal Caliper, Crosswell, UK) in supine position as the distance between the examining table and the apex of the abdominal girth or the largest anteroposterior diameter between the xiphoid process and the umbilicus.

\section{Assessment of metabolic traits}

Systolic and diastolic blood pressure was measured by using an electronic blood pressure meter (OSZ 5 easy, Welch Allyn, Jungingen, Germany) with the subject being in a seated position for at least 5 min. Blood samples for the determination of metabolic markers were drawn in all the patients in the morning between 08:00 and 11:00 h after an overnight fast. The following metabolic markers were determined: Serum concentrations of glucose, insulin, triglycerides (TG), total cholesterol (Chol), low- and high-density lipoprotein (LDL and HDL), and uric acid. The Chol/HDL ratio was calculated [32].

Insulin was measured by chemiluminescence immunoassay (Beckman Coulter International S.A., Nyon, Switzerland) with a CV of less than $10 \%$. Glycolized hemoglobin levels (HbA1c) were assessed in whole blood samples by immunoassay (DCA Systems, Siemens Healthcare Diagnostics Inc., New York, USA). The remaining laboratory blood variables were assayed by clinical routine methods by the local hospital laboratory (Zentrum für Labormedizin, St. Gallen, Switzerland). Homeostasis model assessment (HOMA-IR) was taken as an estimate of insulin resistance [32] and was computed upon the formula: HOMA-IR = (fasting insulin $(\mathrm{mU} / \mathrm{I}) \times$ fasting glucose $(\mathrm{mmol} / \mathrm{l})) / 22.5$.

\section{Statistical analysis}

Data are presented as mean \pm SD and range. All values were tested for normal distribution by using the Kolmogorov-Smirnov test. In case of non-normal distribution respective data were logarithmized (log). For analysis of blood pressure data subjects taking antihypertensive drugs ( $n=86$ ) were excluded; for analysis of glucose metabolism related traits subjects taking anti-diabetic drugs $(n=21)$ were excluded; and for analysis of lipid levels patients taking lipidlowering drugs $(n=25)$ were excluded.

Differences between women and men were assessed by using unpaired Student's t tests. Bivariate association between anthropometric variables and metabolic traits was evaluated by Pearson's correla- 
tion analyses. Multiple stepwise linear regression analysis was performed to test for the independence of associations. $R^{2}$ values obtained from respective regression models were taken as measures of the amount of explained variance in the respective dependent variable (here metabolic traits) upon the considered independent variable (here anthropometric measures) and expressed as \%. Multivariate models included age, body weight or BMI, SAD, WC, WHR and W/Ht ratio as independent variables, whereas variables of metabolic traits were set as dependent variables. Variables that include the same measures, e. g., BMI and W/Ht ratio or WC and WHR, were not computed in the same model. A P value of less than 0.05 was considered significant.

\section{Results}

\section{Subjects' characteristics}

Characteristics including anthropometric measures of the subjects are provided in $>$ Table $\mathbf{1}$ and data on blood pressure and metabolic blood markers in > Table 2. Of note, men were older, taller, heavier and had higher BMI, SAD, WC, WHR, W/Ht, systolic and diastolic blood pressure, insulin, HOMA-IR, TG, Chol/HDL ratio, and uric acid levels than women (all $p<0.04$ ).

\section{Correlation analyses}

Results of correlation analyses between anthropometric variables and metabolic traits are provided in $>$ Table $\mathbf{3}$ for women and in - Table 4 for men.

In women, HbA1c levels, serum glucose and insulin concentrations, as well as HOMA-IR were correlated with all anthropometric measures except for WHR (all $p<0.05$ ). TG levels were correlated with SAD, WC, WHR, and W/Ht (all p<0.02). Total Chol was inversely correlated with SAD and BMI (all $p<0.04$ ) while LDL was not correlated with any of the anthropometric variables (all $p>0.24$ ). HDL was correlated inversely with WC and W/Ht (all p <0.05) while Chol/ HDL positively correlated with WC and WHR (both $p<0.04$ ). Serum uric acid levels significantly correlated with all anthropometric parameters (all $p<0.001$ ) expect for WHR $(p=0.14)$.

In men, metabolic traits in general appeared to be much weaker correlated with anthropometric measures than in women. Serum glucose levels were only correlated with $\operatorname{SAD}(p=0.004)$ and total Chol was correlated with WHR $(p=0.02)$. None of the other glucose or lipid metabolism markers correlated with any of the anthropometric measures (all $p>0.19$ ). However, serum uric acid levels were correlated with WC, W/Ht, and BMI (all $\mathrm{p}<0.05$ )

- Table 1 Subjects' characteristics and anthropometric data.

\begin{tabular}{|c|c|c|c|}
\hline & Women N= 204 & Men N= 69 & $\mathbf{P}$ \\
\hline Age (years) & $41.7 \pm 12.8(18-77)$ & $46.2 \pm 12.2(18-75)$ & 0.013 \\
\hline Height $(\mathrm{cm})$ & $164 \pm 7(148-188)$ & $176 \pm 7(160-196)$ & $<0.001$ \\
\hline Weight $(\mathrm{kg})$ & $112 \pm 19(77-169)$ & $140 \pm 23(97-208)$ & $<0.001$ \\
\hline BMI $\left(\mathrm{kg} / \mathrm{m}^{2}\right)$ & $41.5 \pm 6.5(30.1-62.1)$ & $44.9 \pm 6.7(34.2-64.0)$ & $<0.001$ \\
\hline $\mathrm{SAD}(\mathrm{cm})$ & $20.8 \pm 5.3(11.5-36.0)$ & $25.8 \pm 5.4(17.5-36.0)$ & $<0.001$ \\
\hline Waist circumference $(\mathrm{cm})$ & $122 \pm 13(96-168)$ & $140 \pm 15(110-184)$ & $<0.001$ \\
\hline Hip circumference $(\mathrm{cm})$ & $123 \pm 14(92-175)$ & $133 \pm 16(104-189)$ & $<0.001$ \\
\hline WHR $(\mathrm{cm} / \mathrm{cm})$ & $0.9 \pm 0.1(0.7-1.8)$ & $1.1 \pm 0.1(0.9-1.2)$ & $<0.001$ \\
\hline $\mathrm{W} / \mathrm{Ht}(\mathrm{cm} / \mathrm{cm})$ & $0.7 \pm 0.1(0.6-1.0)$ & $0.8 \pm 0.1(0.7-1.0)$ & $<0.001$ \\
\hline
\end{tabular}

- Table 2 Metabolic traits assessed in the obese study population.

\begin{tabular}{|c|c|c|c|c|c|}
\hline & $\mathbf{N}$ & Women & $\mathbf{N}$ & Men & $\mathbf{P}$ \\
\hline 1Systolic blood pressure $(\mathrm{mmHg})$ & 143 & $133 \pm 17(97-204)$ & 44 & $147 \pm 22(89-192)$ & $<0.001$ \\
\hline 1Diastolic blood pressure $(\mathrm{mmHg})$ & 143 & $86 \pm 12(60-127)$ & 44 & $90 \pm 11(60-123)$ & 0.029 \\
\hline${ }^{2 \mathrm{HbA1c}(\%)}$ & 135 & $5.7 \pm 0.7(4.8-10.3)$ & 37 & $5.9 \pm 0.9(4.9-9.3)$ & 0.219 \\
\hline${ }^{2}$ Glucose (mmol/l) & 176 & $5.6 \pm 1.4(4.1-15.1)$ & 50 & $6.1 \pm 2.4(3.9-18.4)$ & 0.226 \\
\hline${ }^{2}$ Insulin (mU/I) & 151 & $15.1 \pm 11.6(2.8-82.2)$ & 42 & $21.5 \pm 15.7(4.7-74.3)$ & 0.017 \\
\hline${ }^{2}$ HOMA-IR & 151 & $3.9 \pm 3.9(0.6-29.2)$ & 42 & $5.5 \pm 4.6(0.8-22.8)$ & 0.023 \\
\hline${ }^{3} \mathrm{TG}(\mathrm{mmol} / \mathrm{l})$ & 159 & $1.7 \pm 1.3(0.4-6.8)$ & 43 & $2.3 \pm 1.4(0.4-6.2)$ & 0.006 \\
\hline${ }^{3}$ Cholesterol (mmol/l) & 190 & $5.2 \pm 1.0(2.8-9.1)$ & 58 & $5.3 \pm 0.9(3.2-7.2)$ & 0.54 \\
\hline${ }^{3} \mathrm{LDL}(\mathrm{mmol} / \mathrm{l})$ & 155 & $3.3 \pm 0.9(1.2-6.2)$ & 40 & $3.1 \pm 1.0(0.4-5.0)$ & 0.192 \\
\hline${ }^{3} \mathrm{HDL}(\mathrm{mmol} / \mathrm{l})$ & 160 & $1.3 \pm 0.4(0.7-3.2)$ & 44 & $1.2 \pm 0.8(0.6-5.6)$ & 0.256 \\
\hline${ }^{3}$ Chol/HDL (mmol/l) & 160 & $4.2 \pm 1.3(1.6-8.3)$ & 43 & $5.0 \pm 1.7(0.8-8.6)$ & 0.004 \\
\hline Uric acid (mmol/l) & 165 & $336 \pm 86(149-662)$ & 55 & $411 \pm 92(226-629)$ & $<0.001$ \\
\hline \multicolumn{6}{|c|}{$\begin{array}{l}\text { All values are mean } \pm \text { SD (range); }{ }^{1} \text { Patients taking hypertensive-lowering drugs were excluded from analyses }{ }^{2} \text { Patients taking antidiabetic-lowering } \\
\text { drugs were excluded from analyses }{ }^{3} \text { Patients taking lipid-lowering drugs were excluded from analyses. HOMA-IR: Homeostatic Model Assessment of } \\
\text { insulin resistance; TG: Triglycerides; HDL and LDL: low and high density lipoprotein cholesterol }\end{array}$} \\
\hline
\end{tabular}


- Table 3 Bivariate correlations between anthropometric variables and metabolic traits in women.

\begin{tabular}{|c|c|c|c|c|c|}
\hline & SAD & Waist & WHR & $\mathbf{W} / \mathrm{Ht}$ & BMI \\
\hline 1Systolic blood pressure & 0.13 & 0.11 & 0.11 & 0.11 & 0.11 \\
\hline 1Diastolic blood pressure & 0.07 & 0.07 & 0.16 & 0.03 & -0.04 \\
\hline${ }^{2 \mathrm{HbA1c}}$ & $0.31^{* * *}$ & $0.34^{* * *}$ & $0.17^{*}$ & $0.31^{* * *}$ & $0.19^{*}$ \\
\hline${ }^{2}$ Glucose & $0.22 * *$ & $0.28 * * *$ & 0.05 & $0.25 * *$ & $0.15^{*}$ \\
\hline Insulin & $0.40 * * *$ & $0.38 * * *$ & 0.08 & $0.33^{* * *}$ & $0.39 * * *$ \\
\hline 2HOMA-IR & $0.29 * * *$ & $0.40 * * *$ & 0.12 & $0.36 * * *$ & $0.34 * * *$ \\
\hline${ }^{3} \mathrm{TG}$ & $0.19^{*}$ & $0.22 * *$ & $0.31^{* *}$ & $0.19^{*}$ & 0.01 \\
\hline${ }^{3}$ Cholesterol & $-0.23 * *$ & -0.08 & 0.08 & -0.03 & $-0.18^{*}$ \\
\hline${ }^{3} \mathrm{LDL}$ & -0.09 & 0.001 & 0.01 & 0.02 & -0.08 \\
\hline${ }^{3} \mathrm{HDL}$ & -0.15 & $-0.27^{* *}$ & -0.11 & $-0.17^{*}$ & -0.15 \\
\hline${ }^{3}$ Chol/HDL & -0.002 & $0.20^{*}$ & $0.17^{*}$ & 0.14 & 0.02 \\
\hline Uric acid & $0.39 * * *$ & $0.40 * * *$ & 0.12 & $0.36^{* * *}$ & $0.34^{* * *}$ \\
\hline
\end{tabular}

${ }^{*} \mathrm{P}<0.05 * * \mathrm{P}<0.01 * * * \mathrm{P}<0.001$; 1 Patients taking hypertensive-lowering drugs were excluded from analyses 2Patients taking antidiabetic-lowering drugs were excluded from analyses ${ }^{3}$ Patients taking lipid-lowering drugs were excluded from analyses. HOMA-IR: Homeostatic Model Assessment of insulin resistance; TG: Triglycerides; HDL and LDL: low and high density lipoprotein cholesterol

- Table 4 Bivariate correlations between anthropometric variables and metabolic traits in men.

\begin{tabular}{|c|c|c|c|c|c|}
\hline & SAD & Waist & WHR & $\mathbf{W} / \mathrm{Ht}$ & BMI \\
\hline 1Systolic blood pressure & 0.09 & -0.10 & 0.01 & -0.16 & -0.03 \\
\hline 1'Diastolic blood pressure & 0.12 & -0.12 & -0.05 & -0.15 & -0.05 \\
\hline${ }^{2} \mathrm{HbA1c}$ & 0.02 & -0.17 & 0.23 & -0.04 & -0.12 \\
\hline${ }^{2}$ Glucose & $0.39 * *$ & 0.08 & -0.04 & 0.17 & 0.13 \\
\hline 2Insulin & 0.12 & 0.22 & 0.22 & 0.25 & 0.28 \\
\hline${ }^{2} \mathrm{HOMA}-\mathrm{IR}$ & 0.19 & 0.20 & 0.21 & 0.25 & 0.28 \\
\hline${ }^{3} \mathrm{TG}$ & 0.13 & -0.01 & 0.07 & -0.06 & -0.03 \\
\hline${ }^{3}$ Cholesterol & -0.16 & -0.03 & $-0.31^{*}$ & -0.04 & -0.03 \\
\hline${ }^{3} \mathrm{LDL}$ & -0.14 & -0.14 & -0.16 & -0.17 & -0.16 \\
\hline${ }^{3} \mathrm{HDL}$ & 0.17 & -0.14 & -0.008 & -0.12 & -0.09 \\
\hline${ }^{3}$ Chol/HDL & -0.20 & 0.12 & -0.18 & 0.07 & 0.08 \\
\hline Uric acid & 0.26 & $0.39 * *$ & -0.04 & $0.32^{*}$ & $0.27^{*}$ \\
\hline
\end{tabular}

\section{Multivariate regression analyses on each abdominal obesity marker}

Fig. 1 illustrated the strength of association (i. e., $\mathrm{R}^{2}=$ explained variance) between metabolic variables and each anthropometric abdominal obesity marker adjusted for age and BMI (SAD, WC, WHR) or body weight $(\mathrm{W} / \mathrm{Ht})$ as revealed by multiple linear regression analysis. For comparison, the association of metabolic variables with BMI adjusted for age is also provided. As can be seen, women overall showed much more associations than men. Systolic and diastolic blood pressure as well as LDL values were not associated with any anthropometric parameters in both sexes and thus, are not illustrated.

In women, serum glucose levels were, independently of age and $\mathrm{BMI} /$ body weight, associated with $\operatorname{SAD}\left(\mathrm{R}^{2}=4.1 \%\right), \mathrm{WC}\left(\mathrm{R}^{2}=6.8 \%\right)$, $\mathrm{W} / \mathrm{Ht}\left(R^{2}=4.8 \%\right)$, and also with $B M I$ independently of age $\left(R^{2}=3.4 \%\right)$. In men, glucose levels were only associated with SAD $\left(R^{2}=15.7 \%\right)$. Serum insulin levels were independently associated with SAD $\left(R^{2}=16.1 \%\right)$, WC $\left(R^{2}=2.6 \%\right)$, WHR $\left(R^{2}=3.8 \%\right)$, and BMI $\left(R^{2}=15.6 \%\right)$ in women but not with any anthropometric measures in men. HbA1c, HOMA-IR, and TG levels were associated with all anthropometric markers of abdominal obesity (SAD: $\mathrm{R}^{2}=8.5 \%$; $18.1 \%$; $5.4 \%$; respectively, WC: $R^{2}=11.4 \%$; $18.7 \%$; $4.8 \%$; respectively, WHR: $\mathrm{R}^{2}=3.5 \%$; $5.5 \%$; $9.8 \%$; respectively, W/Ht: $\mathrm{R}^{2}=7.4 \%$; $3.0 \%$; $4.7 \%$; respectively) as well as with $\mathrm{BMI}\left(\mathrm{R}^{2}=4.8 \%\right.$; $16.3 \%$; $3.1 \%$; respectively) in women whereas in men no such associations were found. Total Chol levels were associated with $\operatorname{SAD}\left(R^{2}=5.4 \%\right)$ and $B M I\left(R^{2}=3.3 \%\right)$ in women and with WHR $\left(R^{2}=9.6 \%\right)$ in men. In women, HDL was associated with SAD $\left(R^{2}=2.9 \%\right)$ and WC $\left(R^{2}=7.3 \%\right)$ and Chol/HDL with WC $\left(R^{2}=4.0 \%\right)$ and WHR $\left(R^{2}=3.0 \%\right)$. In men, no such associations were found. Serum uric acid levels were independently associated with SAD $\left(R^{2}=15.1 \%\right)$, WC $\left(R^{2}=16.3 \%\right), \operatorname{WHR}\left(R^{2}=5.1 \%\right)$, and $B M I\left(R^{2}=11.6 \%\right)$ in women and with WC $\left(R^{2}=15.3 \%\right)$ and $B M I\left(R^{2}=7.3 \%\right)$ in men. 

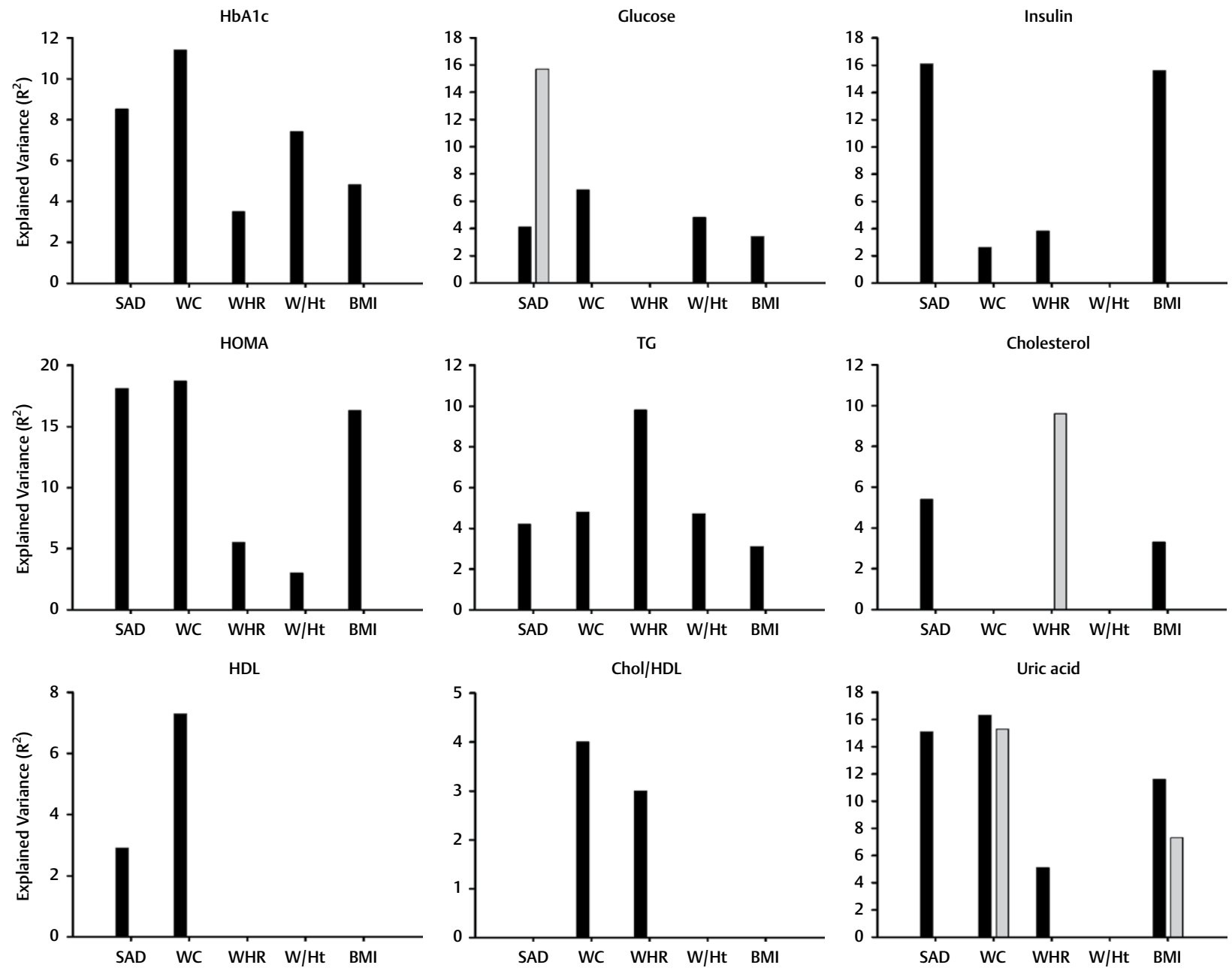

- Fig. 1 Explained variance $\left(R^{2}\right)$ of the metabolic traits upon distinct anthropometric indices. Men are grey, women are black colums; SAD, WC, WHR - related models included age and BMI as independent variables; $\mathrm{W} / \mathrm{Ht}$ related models included age and weight as independent variables; $\mathrm{BMI}$ related models included only age as independent variables. HOMA: Homeostatic Model Assessment of insulin resistance; TG: Triglycerides; HDL: high density lipoprotein cholesterol.

\section{Comparison of strength of associations between different abdominal obesity markers}

To compare the strength of associations of distinct abdominal obesity markers with each metabolic variable all independent anthropometric variables were included in the respective multiple stepwise regression model at the same time. For women, results of these analyses are provided in $>$ Table 5 .

In women, age was the only predictor of systolic blood pressure and the same was true for serum LDL levels. While age was also a strong predictor of $\mathrm{HbA} 1 \mathrm{c}$ and serum glucose levels, measures of abdominal obesity additionally explained some of the variance of these glucose metabolism parameters. For both, fasting glucose and $\mathrm{HbA} 1 \mathrm{c}$, WC was a stronger predictor than SAD. Serum insulin levels were best predicted by SAD but, of note, their prediction was improved further when WC or WHR were additionally included in the respective model. HOMA-IR values were best predicted by WC followed by SAD. Again, prediction of HOMA-IR was even improved when SAD and WC or WHR were included in the same model. Serum TG levels were best predicted by WHR followed by WC and W/Ht. However, inclusion of SAD in the respective WC and WHR model further improved the prediction. SAD was the only anthropometric measure to predict total Chol levels and the association was inverse. Serum HDL levels were best predicted by WC followed by SAD. The Chol/HDL ratio was only predicted by WC and, to a slightly weaker extent, by WHR. Serum uric acid levels were best predicted by WC followed by SAD and then W/Ht. Again, the combination of WC with SAD further improved the prediction of serum uric acid levels.

In men, serum glucose levels were predicted only by SAD $\left(R^{2}\right.$ : $15.7 \%$; beta $=0.37 ; p=0.005)$ while total Chol levels were predicted by WHR ( $R^{2}: 9.6 \%$; beta $\left.=-0.31 ; p=0.02\right)$. Serum uric acid levels were most strongly associated with $W C\left(R^{2}: 15.3 \%\right.$; beta $=0.39$; $p=0.003)$ followed by BMI $\left(R^{2}: 7.3 \%\right.$; beta $\left.=0.27 ; p=0.05\right)$. 


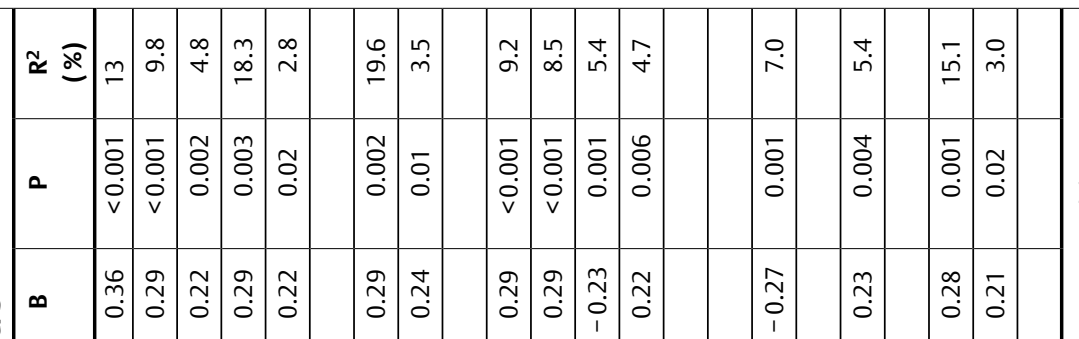

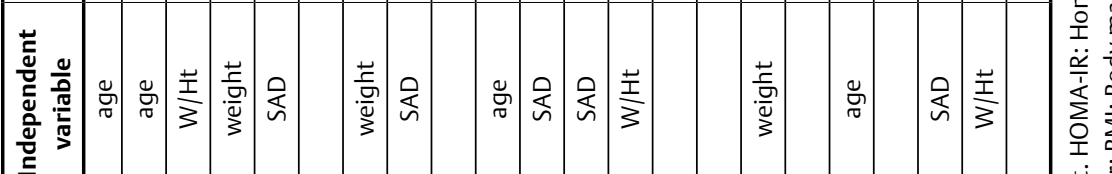

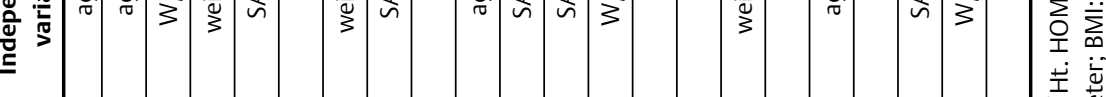

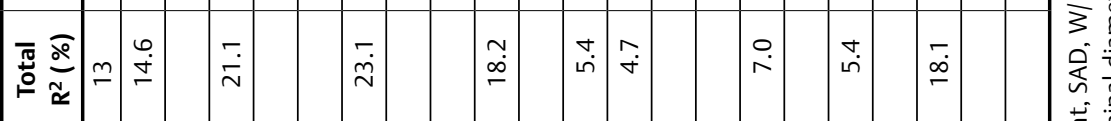

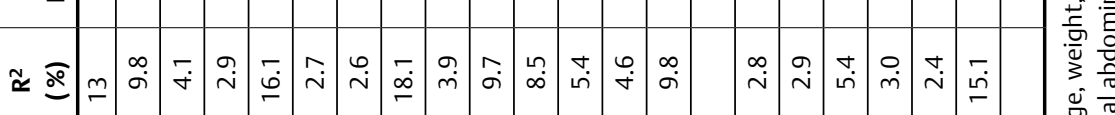

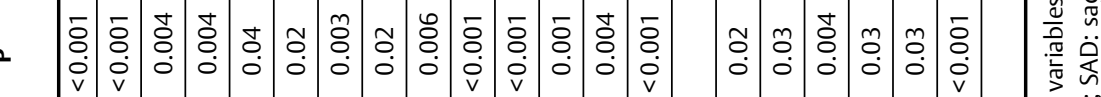

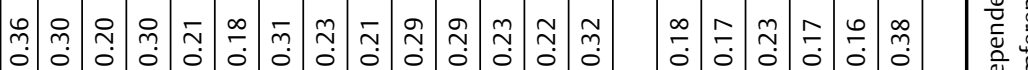

$\frac{\pi}{\frac{2}{2}}$

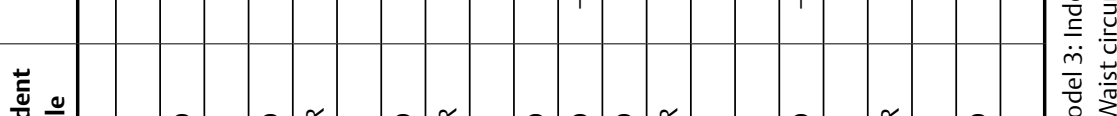

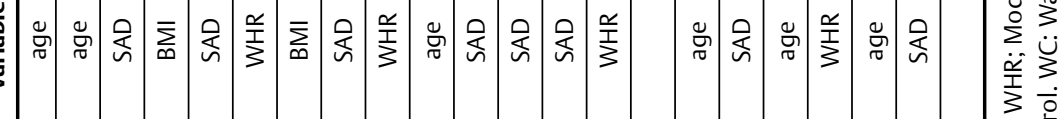

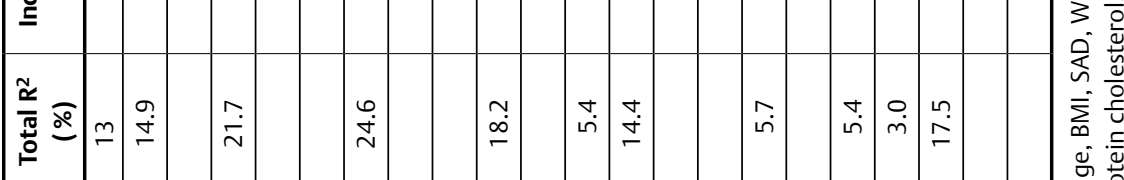

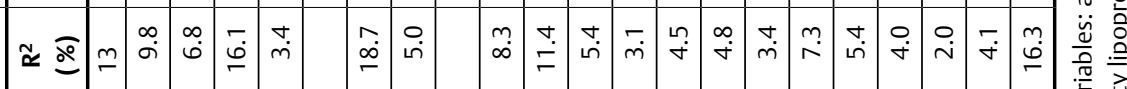

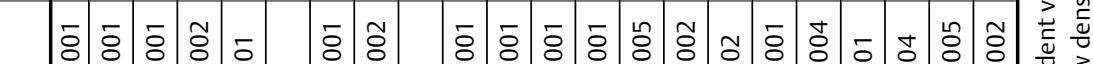

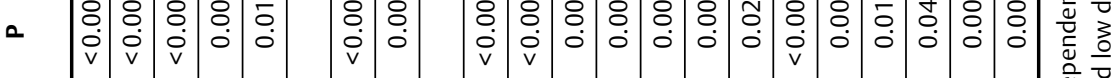

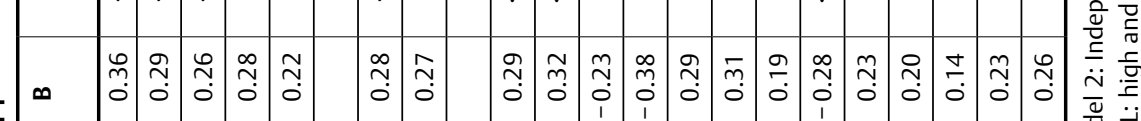

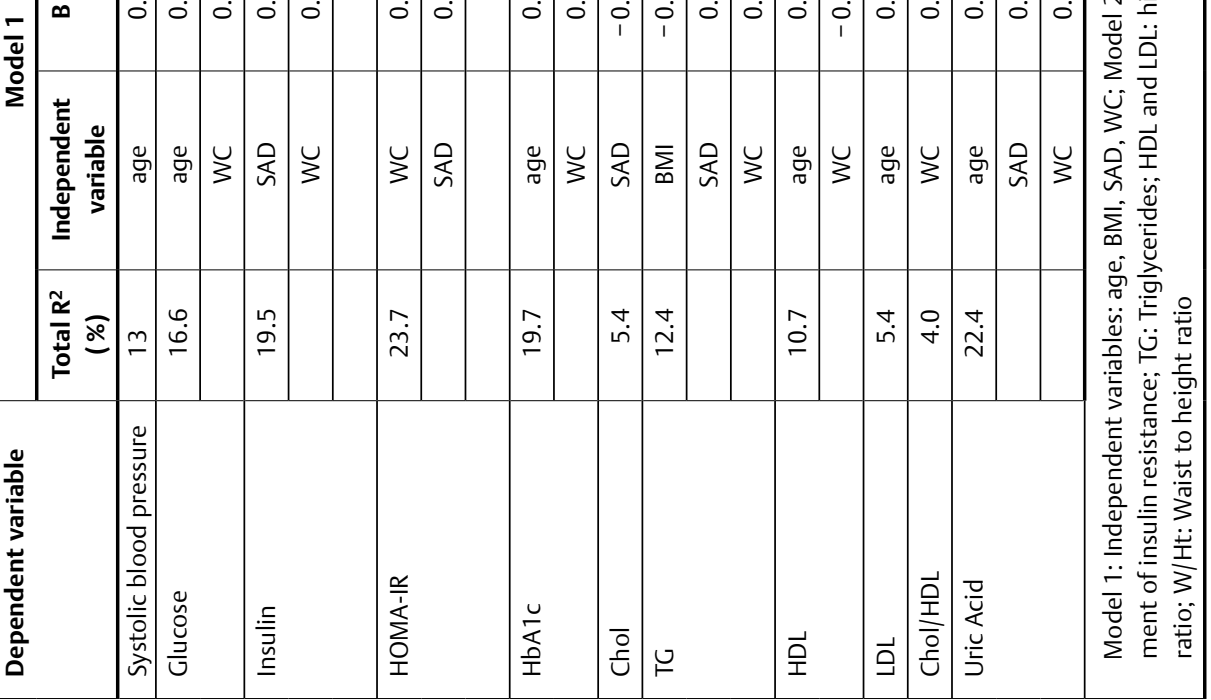




\section{Discussion and Conclusions}

Data of our study confirm the previously observed [15-20] association of WC-related measures of abdominal obesity with metabolic traits in obese subjects independently of BMI as an estimate of general obesity. Our hypothesis that SAD provides a better prediction of metabolic traits in obese subjects than WC-related measures was not confirmed since competitive inclusion of respective variables in multivariate regression analysis revealed an inconsistent picture where in most models WC predicted metabolic traits better than SAD. Overall, the association between anthropometric measures of abdominal obesity and metabolic traits appeared to be stronger in obese women than men. While this finding confirms our previous observations in a larger, independent sample of obese subjects [20] the direct comparison of the association of SAD and WC-related measures with metabolic traits represents the novel and thus, most important aspect of our present study.

In obese women, anthropometric measures of abdominal obesity explained about 4.2 to $15.6 \%$ of the variability in circulating serum insulin, TG, and uric acid levels as well as about $18.7 \%$ of variability in HOMA-IR independently of BMI. This finding supports the previous notion that abdominal obesity is a major driver for insulin resistance and associated metabolic features $[33,34]$ and suggests that this is also true in women who already display a general obesity as indicated by an BMl above $30 \mathrm{~kg} / \mathrm{m}^{2}$.

In obese men, the prediction of metabolic traits upon abdominal obesity measures on top of BMI did not appear to improve as much as in women. It might be argued that this finding was biased by the lower number of men than women included in our study which clearly limited the statistical power to detect significant associations. However, a similar sexual dimorphism in associations was found in our foregoing study that included an independent sample of 241 men with grade 2 or higher obesity [20]. It is well known that men predominantly deposit excess energy in visceral fat stores whereas women usually show a wider distribution of fat accumulation [35]. On this background, we speculate that the greater variability in visceral fat accumulation in obese women than in obese men explains the closer association of abdominal obesity measures with metabolic traits in women.

SAD was not superior in predicting metabolic traits in obese women as compared with WC-related measures in our sample of subjects with a wide range of obesity. This finding contrasts previous findings in overweight and moderately obese men where SAD explained the largest degree of variation in insulin sensitivity compared with other anthropometric measures [36]. Also, analyses on data of the National Health and Nutrition Examination Survey revealed SAD to better predict dysglycemia than WC [37] and a similar observation in regard of the prediction of insulin resistance was made in a Brazilian, mainly non-obese, female study population [38]. Taken together, our present and previous findings speak against our initial assumption that SAD is - in particular obese subjects - superior to WC-related measures in predicting metabolic traits.

It is noteworthy, that for some metabolic variables, i. e., insulin, HOMA-IR, TG, and uric acid, the concurrent inclusion of both, SAD and WC-related measures, increased the predictive value of the respective regression model. This finding suggests that the combination of SAD and WC-related measures may provide a more accurate estimate of visceral adiposity which promotes the associated metabolic alterations. This notion is supported by a previous study in which the combination SAD and WC showed the most accurate prediction of abdominal fat distribution as assessed by MRI [39]. Of note, other anthropometric measures like the neck circumference, not reflecting abdominal obesity, might further improve the prediction of metabolic alterations as has recently been shown in obese women and men [40]. SAD on the other hand has previously been shown to predict the risk of cancer incidence in a non-obese population [41] and also in subjects with severe obesity [42]. Taking together, it appears likely that the combination of distinct anthropometric measures including SAD will provide the most accurate prediction of advance healthy outcomes in obese subjects.

It should be kept in mind that the metabolic consequences of abdominal obesity do not only depend on the extent of visceral fat accumulation but also on specific biological characteristics of visceral fat tissue such as adipocyte sizes and macrophages infiltration $[43,44]$. Also, other factors like cardio-respiratory fitness are well known to modulated the metabolic health state of obese subjects [45]. Therefore, it can be assumed that even the best measure of abdominal obesity will not provide sufficient information on the metabolic health state of a given individual obese subject which makes, in clinical practice, the measurement of relevant metabolic variables in blood samples necessary. However, for epidemiological studies and public health approaches the concurrent assessment of WC and SAD might provide valuable information for metabolic and cardiovascular risk prediction.

Among the tested metabolic traits, uric acid levels showed the most consistent associations with anthropometric measures of abdominal obesity and this was true in both obese women and men. This finding might be of particular interest since it is still not clear whether or not uric acid is causally linked to adverse alteration metabolic traits and cardiovascular disease [46]. A recent study [47] showed that the associations between elevated uric acid levels and markers of altered glucose and lipid metabolism get lost when adjusting for visceral adipose tissue mass as measured by dual-energy X-ray absorptiometry. This finding suggests that visceral obesity represents the link between uric acid and cardio-metabolic disease and our findings may provide further support for this notion.

In summary, our results show that body fat distribution and abdominal obesity in particular play an important role in determining metabolic health in subjects with global obesity. This notion might be particularly true in obese women. While SAD is not superior to WC, the combination of both anthropometric may even increase their predictive value for metabolic traits in obese subjects.

\section{Conflict of Interest}

No conflict of interest has been declared by the authors. 


\section{References}

[1] Despres JP. Body fat distribution and risk of cardiovascular disease: An update. Circulation 2012; 126: 1301-1313

[2] Pischon T, Boeing H, Hoffmann K et al. General and abdominal adiposity and risk of death in Europe. N Engl J Med 2008; 359: 2105-2120

[3] Borel AL, Nazare JA, Smith J et al. Visceral, subcutaneous abdominal adiposity and liver fat content distribution in normal glucose tolerance, impaired fasting glucose and/or impaired glucose tolerance. Int J Obes (Lond) 2015; 39: 495-501

[4] Coutinho T, Goel K, Correa de Sa D et al. Central obesity and survival in subjects with coronary artery disease: a systematic review of the literature and collaborative analysis with individual subject data. J Am Coll Cardiol 2011; 57: 1877-1886

[5] Dallongeville J, Bhatt DL, Steg PH et al. Relation between body mass index, waist circumference, and cardiovascular outcomes in 19,579 diabetic patients with established vascular disease: The Reach Registry. Eur J Prev Cardiol 2012; 19: 241-249

[6] Zeller M, Steg PG, Ravisy J et al. Relation between body mass index, waist circumference, and death after acute myocardial infarction. Circulation 2008; 118: 482-490

[7] Naboush A, Hamdy O. Measuring visceral and hepatic fat in clinical practice and clinical research. Endocrine practice. Endocr Pract 2013; 19: 587-589

[8] Report of a WHO expert consultation. Waist circumference and waist-hip ratio. Geneva, 8-11 December 2008: 1-39

[9] Jacobs E], Newton CC, Wang $\mathrm{Y}$ et al. Waist circumference and all-cause mortality in a large US cohort. Intern Med 2010; 170: 1293-1301

[10] Leitzmann MF, Moore SC, Koster A et al. Waist circumference as compared with body-mass index in predicting mortality from specific causes. PloS one 2011; 6: e18582

[11] Guallar-Castillon P, Balboa-Castillo T, Lopez-Garcia E et al. BMI, waist circumference, and mortality according to health status in the older adult population of Spain. Obesity (Silver Spring) 2009; 17: 2232 2238

[12] Bajaj HS, Brennan DM, Hoogwerf BJ et al. Clinical utility of waist circumference in predicting all-cause mortality in a preventive cardiology clinic population: a PreCIS Database Study. Obesity (Silver Spring) 2009; 17: 1615-1620

[13] Kanaya AM, Vittinghoff E, Shlipak MG et al. Association of total and central obesity with mortality in postmenopausal women with coronary heart disease. Am J Epidemiol 2003; 158: 1161-1170

[14] Bigaard J, Tjonneland A, Thomsen BL et al. Waist circumference, BMI, smoking, and mortality in middle-aged men and women. Obes Res 2003; 11: 895-903

[15] Ashwell M, Gunn P, Gibson S. Waist-to-height ratio is a better screening tool than waist circumference and BMI for adult cardiometabolic risk factors: Systematic review and meta-analysis. Obes Rev 2012; 13: 275-286

[16] Ho SY, Lam TH, Janus ED. Waist to stature ratio is more strongly associated with cardiovascular risk factors than other simple anthropometric indices. Ann Epidemiol 2003; 13: 683-691

[17] Hsieh SD, Yoshinaga H, Muto T. Waist-to-height ratio, a simple and practical index for assessing central fat distribution and metabolic risk in Japanese men and women. Int J Obes Relat Metab Disord 2003; 27: 610-616

[18] Brenner DR, Tepylo K, Eny KM et al. Comparison of body mass index and waist circumference as predictors of cardiometabolic health in a population of young Canadian adults. Diabetol Metab Syndr 2010; 2: 28
[19] Bener A, Yousafzai MT, Darwish S et al. Obesity index that better predict metabolic syndrome: Body mass index, waist circumference, waist hip ratio, or waist height ratio. J Obesity 2013; 2013: 269038

[20] Zazai R, Wilms B, Ernst B et al. Waist circumference and related anthropometric indices are associated with metabolic traits in severely obese subjects. Obes Surg 2014; 24: 777-782

[21] Radholm K, Tengblad A, Dahlen E et al. The impact of using sagittal abdominal diameter to predict major cardiovascular events in European patients with type 2 diabetes. Nutrition, metabolism, and cardiovascular diseases. Nutr Metab Cardiovasc Dis 2017; 418-422

[22] Vasques AC, Cassani RS, Forti AC et al. Sagittal abdominal diameter as a surrogate marker of insulin resistance in an admixtured population-Brazilian Metabolic Syndrome Study (BRAMS). PloS one 2015; 10: e0125365of a WHO expert consultation

[23] Kahn HS, Gu Q, Bullard KM et al. Population distribution of the sagittal abdominal diameter (SAD) from a representative sample of US adults: comparison of SAD, waist circumference and body mass index for identifying dysglycemia. PloS one 2014; 9: e108707

[24] de Souza NC, de Oliveira EP. Sagittal abdominal diameter shows better correlation with cardiovascular risk factors than waist circumference and BMI. J Diabetes Metab Disord 2013; 12: 41

[25] Pajunen P, Rissanen H, Laaksonen MA et al. Sagittal abdominal diameter as a new predictor for incident diabetes. Diabetes care 2013; 36: $283-288$

[26] Duarte Pimentel G, Portero-McLellan KC, Maesta N et al. Accuracy of sagittal abdominal diameter as predictor of abdominal fat among Brazilian adults: A comparation with waist circumference. Nutr Hosp 2010; 25: 656-661

[27] Riserus U, Arnlov ], Brismar K et al. Sagittal abdominal diameter is a strong anthropometric marker of insulin resistance and hyperproinsulinemia in obese men. Diabetes Care 2004; 27: 2041-2046

[28] Ohrvall M, Berglund L, Vessby B. Sagittal abdominal diameter compared with other anthropometric measurements in relation to cardiovascular risk. Int J Obes Relat Metab Disord 2000; 24: 497-501

[29] Petersson H, Daryani A, Riserus U. Sagittal abdominal diameter as a marker of inflammation and insulin resistance among immigrant women from the Middle East and native Swedish women: A crosssectional study. Cardiovasc Diabetol 2007; 6: 10

[30] Frenhani PB, Pimentel GD, Portero-McLellan KC et al. Sagittal abdominal diameter as a predictor of visceral abdominal fat, insulin resistance, dyslipidemia and inflammation in overweight Brazilian adults. Clin Nutr Suppl 2008; 3: 126

[31] Yim JY, Kim D, Lim SH et al. Sagittal abdominal diameter is a strong anthropometric measure of visceral adipose tissue in the Asian general population. Diabetes Care 2010; 33: 2665-2670

[32] Matthews DR, Hosker JP, Rudenski AS et al. Homeostasis model assessment: insulin resistance and beta-cell function from fasting plasma glucose and insulin concentrations in man. Diabetologia 1985; 28: $412-419$

[33] Lopes HF, Correa-Giannella ML, Consolim-Colombo FM et al. Visceral adiposity syndrome. Diabetol Metab Syndr 2016; 8: 40

[34] de Oliveira EP, Burini RC. High plasma uric acid concentration: causes and consequences. Diabetol Metab Syndr 2012; 4: 12

[35] Palmer BF, Clegg DJ. The sexual dimorphism of obesity. Mol Cell Endocrinol 2015; 402: 113-119

[36] Riserus U, Ärnlov J, Brismar K et al. Sagittal abdominal diameter is a strong anthropometric marker of insulin resistance and hyperproinsulinemia in obese men. Diabetes Care 2004; 27: 2041-2046

[37] Kahn HS, Gu Q, Bullard KM et al. Population distribution of sagittal abdominal diameter (SAD) from a representative sample of US adults: comparison of SAD, waist circumference and body mass index for identifying dysglycemia. PLoS One 2014; 9: e108707 
[38] Vasques AC, Cassani RS, Forti AC et al. Sagittal abdominal diameter as a surrogate marker of insulin resistance in an admixtured populationBrazilian metabolic syndrome study (BRAMS). PLoS One 2015; 10: e0125365

[39] Lee J], Freeland-Graves JH, Pepper MR et al. Predictive equations for central obesity via anthropometrics, stereovision imaging and MRI in adults. Obesity (Silver Spring) 2014; 22: 852-862

[40] Assoy Y, Gateva A, Tsakova A et al. A comparison of the clinical usefulness of neck circumference and waist circumference in individuals with severe obesity. Endocr Res 2017; 42: 6-14

[41] Oppert JM, Charles MA, Thibult $\mathrm{N}$ et al. Anthropometric estimates of muscle and fat mass in relation to cardiac and cancer mortality in men: the Paris Prospective Study. Am J Clin Nutr 2002; 75: 1107-1113

[42] Sjöström L, Gummesson A, Sjöström CD et al. Effects of bariatric surgery on cancer incidence in obese patients in Sweden (Swedish Obese Subject Study): A prospective, controlled intervention trial. Lancet Oncol 2009; 10: 653-662
[43] Tchernof A, Despres JP. Pathophysiology of human visceral obesity: an update. Physiol Rev 2013; 93: 359-404

[44] Bluher M. Adipose tissue inflammation: A cause or consequence of obesity-related insulin resistance? Clin Sci (Lond) 2016; 130: 1603-1614

[45] Waldburger R, Wilms B, Ernst B et al. Cardio-respiratory fitness is independently associated with cardio-metabolic risk markers in severely obese women. Exp Clin Endocrinol Diabetes 2014; 122: 190-194

[46] Martinez-Quintana E, Tugores A, Rodriguez-Gonzalez F. Serum uric acid levels and cardiovascular disease: The Gordian knot. J Thorac Dis 2016; 8: E1462-E1466

[47] Seyed-Sadjadi N, Berg J, Bilgin AA et al. Visceral fat mass: is It the link between uric acid and diabetes risk? Lipids Health Dis 2017; 16: 142 